\title{
Identification of Location and Timing of Guidance Cues in Sympathetic Preganglionic Axons of the Chick
}

\author{
Joseph W. Yip \\ Department of Physiology, School of Medicine, University of Pittsburgh, Pittsburgh, Pennsylvania 15261
}

\begin{abstract}
The outgrowth of preganglionic axons into the sympathetic trunk of the chick occurs at stages 26-27 (5-51/2 d), beginning with $\mathrm{C} 16$ and $\mathrm{T} 1$ and followed by progressively more caudal spinal cord segments. The direction of preganglionic projections is segment specific, with $\mathrm{T} 1$ preganglionic neurons projecting predominantly in the rostral direction and T4 preganglionic neurons projecting predominantly in the caudal direction. These projections are correct from the outset.

A previous study (Yip, 1987) has shown that removal of the neural crest, precursors of target ganglion cells, did not alter the trajectories of preganglionic axons, indicating that target cues are not required for the guidance of these axons. The present work extends that study and examines, through surgical manipulations of the developing embryo, the location and time of appearance of preganglionic axonal guidance cues. Spinal cord transplantation experiments show that segment-specific patterns of preganglionic outgrowth are determined by the local environment of axonal growth and not by the identity of preganglionic populations. In addition, spinal cord transplants of age-mismatched donors and hosts indicate that the timing of preganglionic outgrowth is also under local environmental control. These findings suggest that preganglionic axonal outgrowth is guided by local cues which may appear in a controlied spatiotemporal manner.
\end{abstract}

The pathways taken by axons towards their targets are highly precise and stereotyped. With few exceptions, these pathways are correct from the outset (see, for example, Landmesser, 1978; Eisen and Westerfield, 1986). While it is generally accepted that axons are responding to guidance cues, the nature of these cues remains unknown. In order to identify the cues underlying axonal guidance, it is important to know where and when such cues are expressed. Evidence from explant studies indicates that a diffusible trophic substance released by neuronal targets may be important in axonal guidance (Lumsden and Davies, 1983, 1986; Dodd and Jessell, 1988). Other studies on axonal guidance have suggested that axons are guided by local environmental cues. For example, when presumptive muscles are removed prior to axonal outgrowth in chick embryos, motor axons were still able to follow appropriate pathways into the limb, despite

\footnotetext{
Received Oct. 5, 1989; revised Feb. 13, 1990; accepted Mar. 20, 1990.

Dedicated to Dr. Viktor Hamburger.

This work was supported by National Institutes of I lealth Grant NS-23916. I thank Eric Frank, Lucia Yip, and Christine Capriotti for critical comments on the manuscript, Bao Qin He for excellent technical assistance, and Tom Harper for photography.

Correspondence should be addressed to Joseph W. Yip at the above address.

Copyright (c) 1990 Society for Neuroscience $0270-6474 / 90 / 072476-09 \$ 03.00 / 0$
}

the absence of their target muscles (Tosney and Landmesser, 1984). In addition, in Drosophila wing disc and grasshopper leg isolated in organ culture, sensory neurons still project in their normal stereotyped patterns (Blair and Palka, 1985; Blair et al., 1985; Lefcort and Bentley, 1987).

Successful in vivo studies of axonal guidance mechanisms require a system where the anatomy is relatively simple, the development is well understood, and the presumptive pathways of growing axons are predictable and accessible for manipulations. Sympathetic preganglionic projections of the chick appear to be well suited for studies of axonal guidance. For example, they project only in rostrocaudal directions in the sympathetic trunk, adjacent to the vertebral column. Their pathways are therefore simple and predictable. The chick embryo has the added advantage of being accessible for experimental manipulations.

In the search for guidance mechanisms underlying sympathetic preganglionic projections, a previous study (Yip, 1987) has shown that target cues are not required for the guidance of these axons. The search for preganglionic axonal guidance cues is continued in the present study. Using heterotopic and heterochronic spinal cord transplantations, I have shown that both the direction and timing of preganglionic outgrowth are influenced by the tissue environment along the route of preganglionic projections. These results provide the basis for further characterization of guidance cues in the projection of sympathetic preganglionic axons.

\section{Materials and Methods \\ The chick sympathetic system}

The sympathetic system of the chick is illustrated in Figure 1. The peripheral division consists of a chain of ganglia along each side of the vertebral column. This chain extends from the cervical throughout the sacral levels and is characterized by the presence of a ganglion at each segmental level. Ganglion cclls arise from the ncural crest (sce LeDouarin, 1982) and are innervated by preganglionic neurons located between the brachial and lumbosacral enlargements of the spinal cord (Terni, 1924; Levi-Montalcini, 1950). Preganglionic neurons cluster around the central canal of the spinal cord; their axons exit in the ventral roots of the corresponding spinal segments and enter into the sympathetic trunk (Langley, 1904; Yip, 1986). Depending on the segmental location of their cell bodies, preganglionic neurons may project rostrally, or caudally, to innervate the chain of ganglia (see below).

\section{Preganglionic axonal tracing}

Patterns of preganglionic projections were determined by ortho- and retrograde HRP labeling techniques. Embryos were dissected and eviscerated in oxygenated Tyrode solution (Landmesser, 1978). A dorsal laminectomy was performed to expose the spinal cord for oxygenation. For orthograde labeling, about $0.2 \mu \mathrm{l}$ of a $30 \%$ HRP solution with $1 \%$ lysolecithin was pressure-injected with a micropipette $(20 \mu \mathrm{m}$ tip diameter) into the spinal cord segment of interest, in the region that is adjacent to the dorsal root ganglia. For retrograde labeling, a similar 


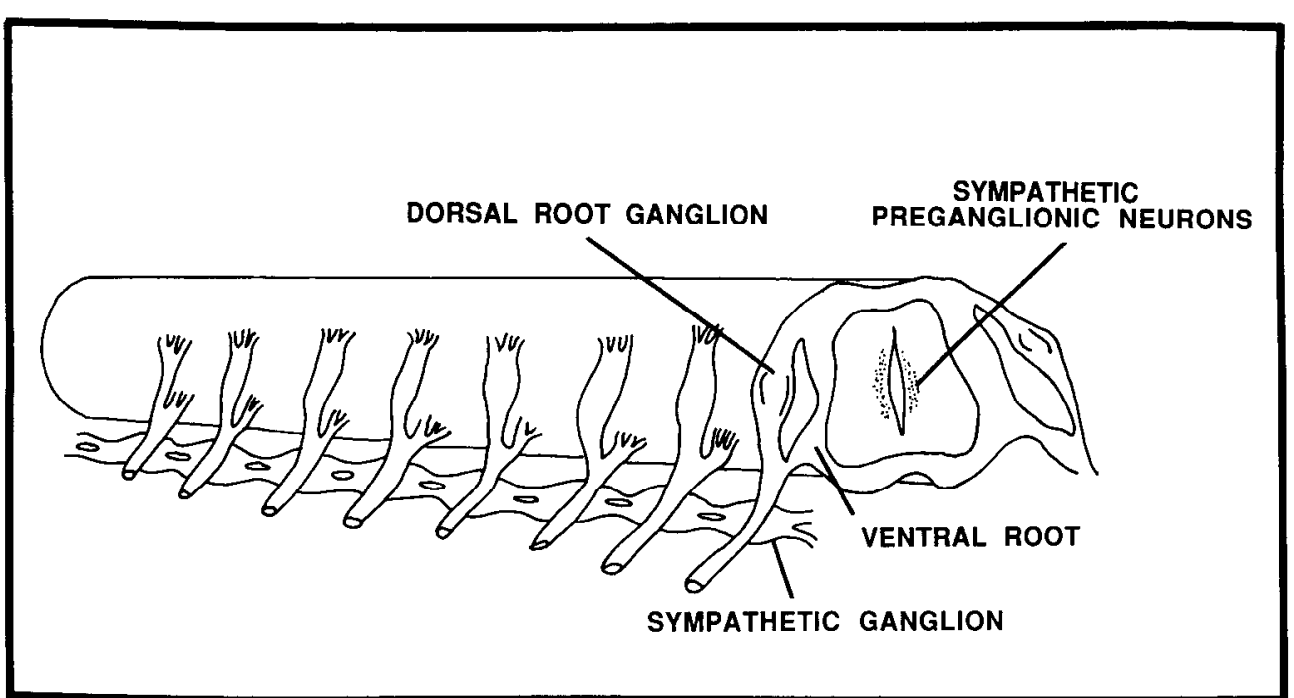

Figure 1. General organization of the chick sympathetic system. Sympathetic ganglia are segmentally arranged along each side of the vertebral column. Ganglion cells are innervated by a column of preganglionic neurons that extends between the brachial and lumbosacral enlargements on each side of the spinal cord. Preganglionic axons exit in the ventral roots to innervate the sympathetic chain ganglia. volume of HRP was injected into the $\mathrm{C} 15$ ganglia. Following HRP injections, the preparation was maintained in an oxygenated culture medium (minimum essential medium, Eagle) at $34^{\circ} \mathrm{C}$ for $5-7 \mathrm{hr}$ to allow for transport of HRP (Yip, 1987). Thereafter, the embryo was fixed for $2 \mathrm{hr}$ with a phosphate-buffered fixative consisting of a mixture of $1 \%$ paraformaldehyde, $2.5 \%$ glutaraldehyde, and $4 \%$ sucrose. At the end of fixation, the embryo was rinsed in phosphate buffer, equilibrated in buffered $30 \%$ sucrose, and then frozen in liquid nitrogen. Serial sections, $30 \mu \mathrm{m}$, in the sagittal or transverse plane were cut with a cryostat. These sections were mounted on gelatin-coated slides and reacted for the presence of HRP using, in most cases, diaminobenzidine (DAB) as the chromagen (Adams, 1977). In some cases, the HRP reaction product was developed in $p$-phenylenediamine and pyrocatechol solution (Hanker et al., 1977). There was no difference in the extent of labeling between these 2 methods.

\section{Experimental manipulations}

Embryos were exposed by opening a window in the shell and stained lightly with a $0.1 \%$ neutral red solution. Levels of the neural tube were determined by counting somites (Levi-Montalcini, 1950). Following manipulation, the window in the shell of the operated embryo was covered with a glass coverslip and sealed with paraffin wax. Operated embryos were then returned to a forced-draft incubator at $37^{\circ} \mathrm{C}$ and $70 \%$ relative humidity and allowed to grow until appropriate stages. All preganglionic projections were determined by orthograde HRP labeling. In order to identify the source and time of appearance of guidance cue for the projection of preganglionic neurons, 3 series of experimental manipulations were performed.

Spinal cord rotations. To examine the relative importance of segmental origins and tissue environmental cues outside of the spinal cord in the projections of preganglionic neurons, the thoracic spinal cord segments T1-T4 were removed from stage 13-14 (48-53 hr) (Hamburger and Hamilton, 1951) embryos with a sharpened tungsten needle, rotated $180^{\circ}$ along the anterior-posterior axis and reimplanted into the same embryo (Fig. 2A). Projections of translocated T1 and T4 preganglionic neurons were examined in stage $30\left(6^{1 / 2-7} \mathrm{~d}\right)$ embryos to determine if they follow pathways that are appropriate to their segmental origin or to their new segmental locations. In addition, the early outgrowth of translocated $\mathrm{T} 1$ and $\mathrm{T} 4$ preganglionic neurons were examined in stage $27\left(5-5^{1 / 2} \mathrm{~d}\right)$ and $28\left(5^{1 / 2}-6 \mathrm{~d}\right)$ embryos to determine if the neurons' local environment could alter the timing of their outgrowth.

Spinal cord transplantations. To examine the projections of preganglionic neurons at spinal levels where they are normally absent, a single segment of the T1 or T4 spinal cord was removed from stage 13-14 embryos and transplanted to the ninth cervical (C9) level of the same embryo, where the corresponding spinal cord segment has been removed. The excised T1 or T4 spinal cord segment was replaced with the C9 spinal cord segment (Fig. $2 B$ ). Projections of translocated T1 and $\mathrm{T} 4$ preganglionic neurons were examined at stage 30 embryos.

Heterochronic spinal cord transplantations. To examine the influence of the tissue environment on the timing of preganglionic outgrowth, a segment of the spinal cord from the T1-T4 spinal levels was removed from older donor embryos (stages 18 and $19,65-72 \mathrm{hr}$ ) and transplanted
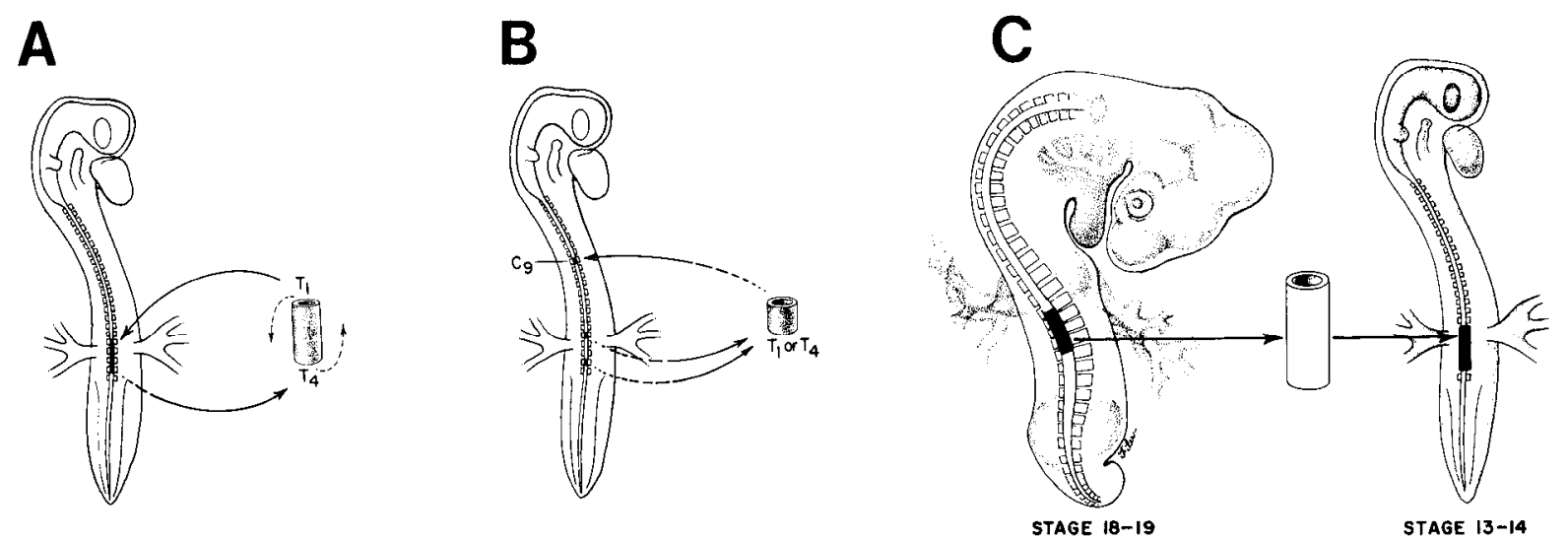

Figure 2. Spinal cord manipulations. $A$, In stage 13-14 embryos, spinal cord segments T1-T 4 were rotated $180^{\circ}$ along the anterior-posterior axis and reimplanted into the same embryos. $B$, In stage 13-14 embryos, single C 9 spinal cord segments were replaced with the T1 or T4 spinal cord segments. $C$, In stage 18-19 embryos, T1-T4 spinal segments were removed and transplanted into stage 13-14 embryos. 
into younger hosts (stages 13 and $14,48-53 \mathrm{hr}$ ) from which an equivalent length of the upper thoracic spinal cord was previously removed (Fig. $2 C)$. T1 and T4 preganglionic outgrowth from donor spinal cords was determined at various stages of host embryos.

\section{Results}

\section{Specificity of preganglionic projections}

The specificity and development of preganglionic projections, reported briefly in a previous study (Yip, 1987), is described in more detail in the present study. Patterns of preganglionic projections are highly stereotyped. Orthograde HRP labeling in 27 embryos at stages $27-32\left(5-7 \frac{1}{2} \mathrm{~d}\right)$ revealed that $\mathrm{T} 1$ preganglionic neurons project predominantly in the rostral direction, whereas T4 preganglionic neurons project predominantly in the caudal direction (Fig. 3). In contrast, orthograde HRP labeling in 28 embryos of the same stages showed that preganglionic neurons arising from the T2 and T3 spinal levels assume more intermediate patterns, with many neurons projecting in the rostral and in the caudal directions. Thus, depending on the segmental level of their cell bodies, preganglionic axons project in highly stereotyped patterns.

Retrograde HRP labeling revealed that each ganglion is supplied by a characteristic set of preganglionic neurons arising from several contiguous spinal cord segments. The $\mathrm{C} 15$ ganglion of stage $32(71 / 2 \mathrm{~d})$ embryos $(n=7)$, for example, is supplied by preganglionic neurons arising from the C16-T3 spinal cord segments. The number of preganglionic neurons supplying this ganglion is greatest from the T1 spinal segment and decreases with distance in more rostral and caudal spinal cord segments (Fig. 4). This pattern of preganglionic projections to the $\mathrm{C} 15$ ganglion of the stage 32 embryo is similar to that found in the adult (Yip, 1986).

\section{Development of preganglionic projections}

Projection of sympathetic preganglionic axons into the sympathetic chain occurs at stages 26-27 (5-51/2 d, 13 embryos), beginning with $\mathrm{C} 16$ and $\mathrm{T} 1$ and followed by progressively more caudal spinal cord segments. Injection of HRP into the T1 and T4 spinal cord segments of stage 27 embryos $(n=5)$ showed a few labeled $\mathrm{T} 1$ axons just entering the sympathetic chain but no T4 axons (Fig. $5 A)$. At stage $28\left(5 \frac{1}{2}-6 \mathrm{~d}, n=10\right)$, T1 preganglionic neurons project 2-3 segments rostrally, whereas T4 axons are just entering the sympathetic chain (Fig. $5 B$ ). By stage $30\left(6^{1 / 2}-7 \mathrm{~d}, n=7\right), \mathrm{T} 1$ axons project at least 4 segments rostrally, and T4 axons project 3 segments caudally (Fig. 3 ).

The direction of preganglionic projections, moreover, is correct from the outset. The predominantly rostral projection of $\mathrm{T} 1$ preganglionic neurons, for example, is apparent as soon as they enter into the sympathetic chain at stage 27 (Fig. 5A). In subsequent stages, more $\mathrm{T} 1$ preganglionic axons are found in the sympathetic chain, but their predominantly rostral projections are maintained (Fig. $5 B$ ).

\section{Direction of preganglionic projections is not intrinsically determined by segmental origins of their cell bodies}

A question that is raised by the stereotyped pathways taken by preganglionic neurons arising from different spinal cord segments is whether the direction of preganglionic projections is intrinsically determined by their segmental origins in the spinal cord. This question was answered by rotation of the T1-T4 spinal cord segment of the stage 13 or 14 embryo $180^{\circ}$ along the A-P axis prior to axonal outgrowth (Fig. $2 A$ ); projections of

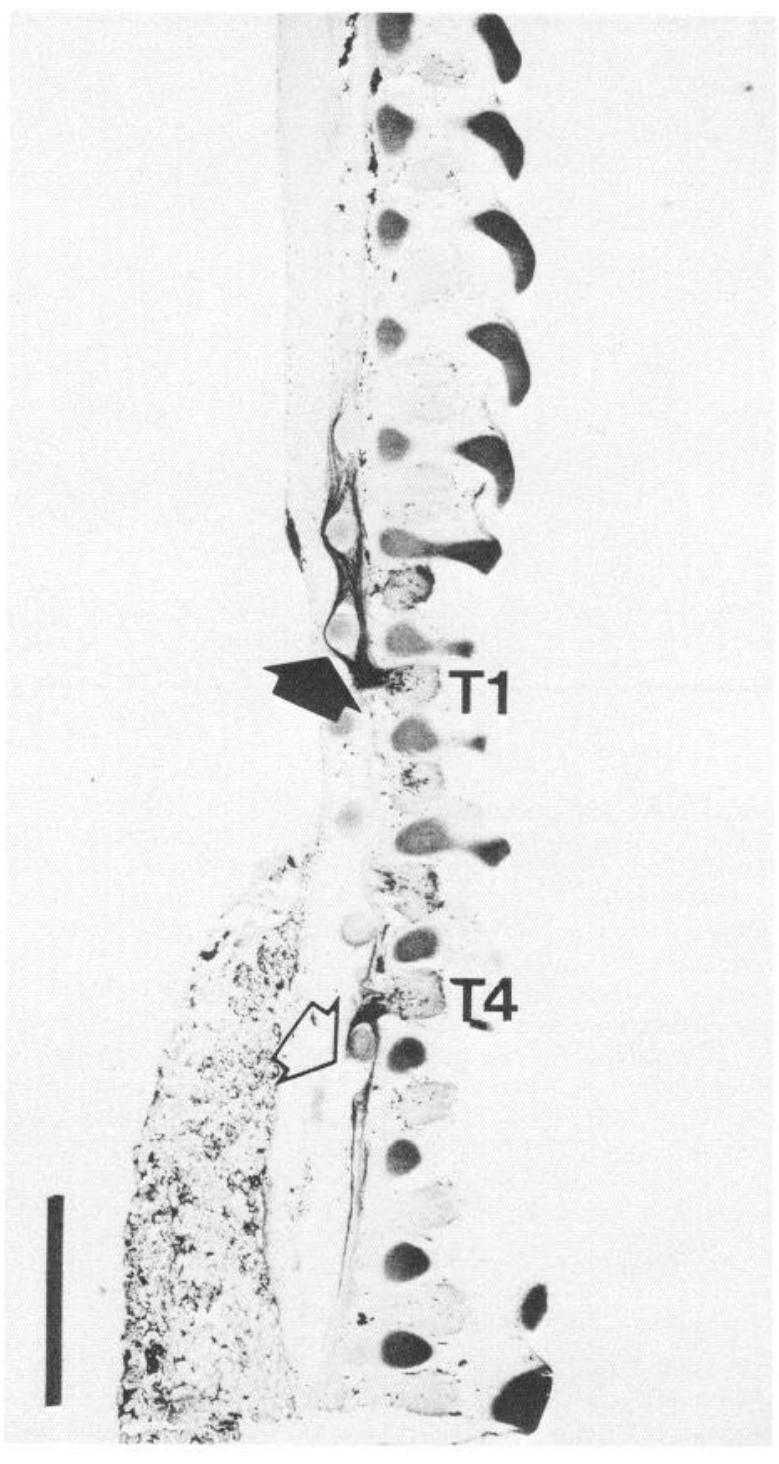

Figure 3. Sagittal section of a stage 30 embryo. Orthograde HRP labeling shows that $\mathrm{T} 1$ preganglionic neurons (closed arrow) project predominantly in the rostral direction, whereas T4 preganglionic neurons (open arrow) project predominantly in the caudal direction. Scale bar, $1 \mathrm{~mm}$.

translocated $\mathrm{T} 1$ and $\mathrm{T} 4$ preganglionic neurons were examined in stage 28 embryos $(n=11)$ to determine if translocated preganglionic neurons project in directions appropriate to their segmental origins or to their new segmental locations. My results indicate that translocated preganglionic neurons do not project in directions appropriate to their segmental origins, but project instead in directions that are appropriate to neurons of their new segmental locations. Thus, translocated T4 preganglionic neurons, which are now located rostrally, project rostrally, and translocated $\mathrm{T} 1$ preganglionic neurons, which are now located caudally, project caudally (Fig. 6). These results indicate that the directions of preganglion projections are not intrinsically determined by their segmental origins in the spinal cord, but appear to depend on factors in the tissue environment outside of the spinal cord. 

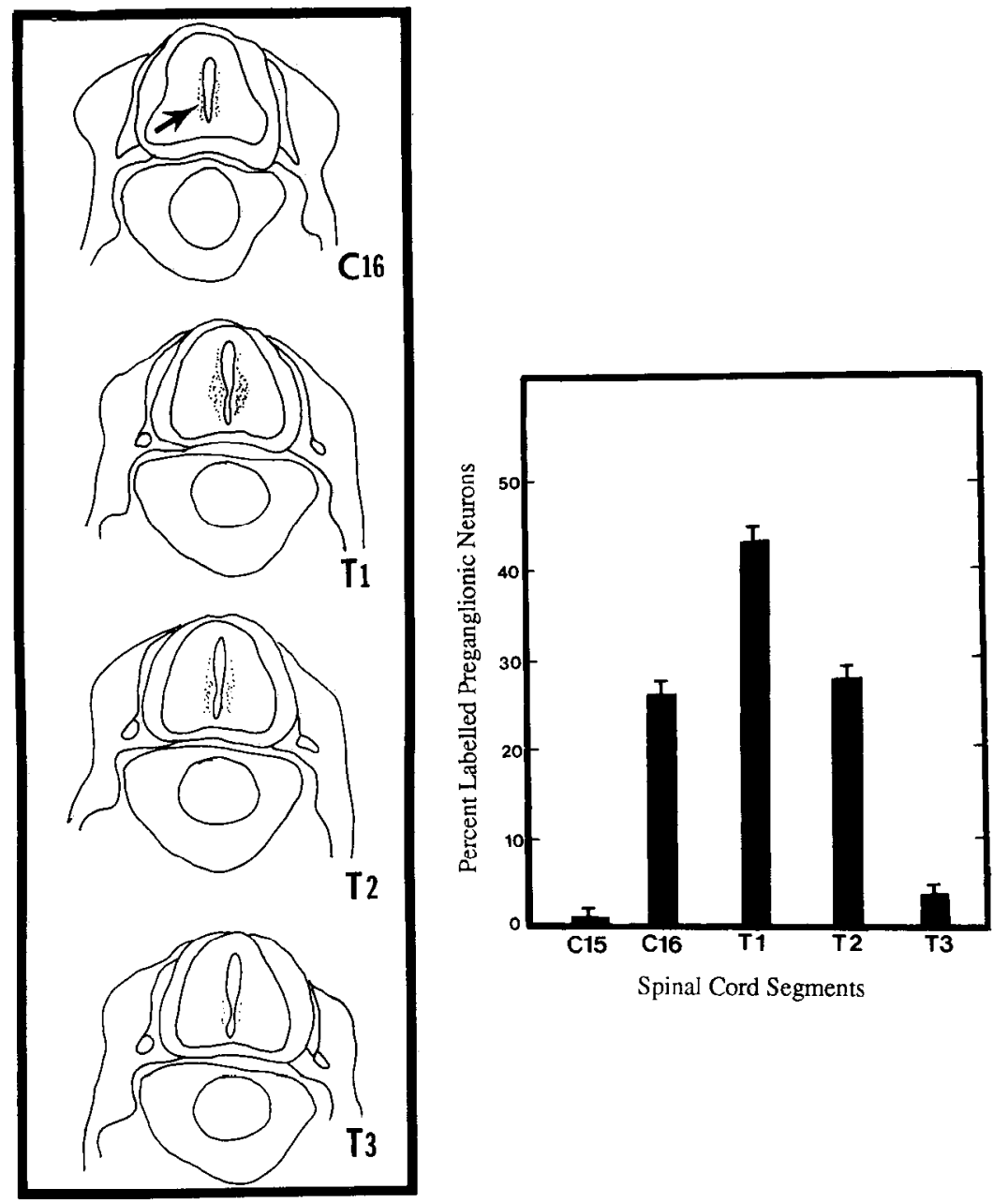

Figure 4. Characteristic pattcrn of preganglionic axons projecting to the C15 ganglia of stage $32(71 / 2 \mathrm{~d})$ embryos as determined by retrograde labeling with HRP injected into the C15 ganglia. Many preganglionic axons in the spinal cord are labeled. The camera lucida drawings on the left show labeled preganglionic neurons (arrow) in spinal cord segments C16-T3. The histogram on the right shows the distribution of labeled neurons within these segments.

Tissue environment outside of spinal cord influences direction of preganglionic projections

In order to further examine the relative importance of segmental origin and tissue environmental cues outside of the spinal cord in the projection of preganglionic neurons, I have transplanted preganglionic neurons to a spinal level where they are normally absent. Thus, a single segment of the $\mathrm{T} 1$ or $\mathrm{T} 4$ spinal cord was removed from stage 13 or 14 embryos and transplanted to the ninth cervical (C9) level, where a corresponding spinal cord segment had been removed (Fig. $2 B$ ). The projections of T1 (24 embryos) or T4 (26 embryos) preganglionic neurons in their novel environment were examined in stage 30 embryos with orthograde HRP labeling. My results indicate that both $\mathrm{T} 1$ and T4 preganglionic neurons transplanted to the C9 level project more or less equally in both directions, instead of their normal, predominantly rostral or caudal direction. In the stage $30 \mathrm{em}-$ bryo, they project, on average, 4 segments rostrally and 4 segments caudally (Fig. 7). These results clearly demonstrate that the directions of preganglionic projections are not determined by their initial segmental origins in the spinal cord but are influenced by the tissue environment outside of the spinal cord.

\section{Tissue environment influences the timing of preganglionic} outgrowth

Since there is a rostral to caudal sequence in the normal outgrowth of sympathetic preganglionic axons, a question that aris- es is whether the tissue environment outside of the spinal cord also influences the timing of preganglionic axonal outgrowth. In order to answer this question, I examined whether T4 neurons in embryos which had the T1-T4 spinal cord segment rotated $180^{\circ}$ along the A-P axis would now project ahead of $\mathrm{T} 1$ neurons. My results indicate that they do. When T4 preganglionic neurons were translocated to the $\mathrm{T} 1$ spinal level, they project according to the schedule of normal $\mathrm{T} 1$ preganglionic neurons, that is, they extend their axons into the sympathetic trunk at stage 27 (Fig. $8 A$ ), ahead of those of translocated $\mathrm{T} 1$ preganglionic neurons ( 9 embryos). By stage 28 (11 embryos), translocated T4 preganglionic neurons extend 3 segments into the sympathetic trunk (Fig. $8 B$ ). Conversely, $\mathrm{T} 1$ preganglionic neurons translocated to the T4 segmental level project according to the schedule that is appropriate to $\mathrm{T} 4$ preganglionic neurons, that is, they enter into the sympathetic chain at stage 28 and project 3 segments caudally by stage 30 ( 20 embryos).

In order to further examine the influence of the tissue environment on the timing of preganglionic axonal outgrowth, spinal cords from older embryos (stages 18-19) were transplanted into younger hosts (stages 13-14) to determine whether preganglionic neurons from donor spinal cord would project according to the schedule of the host (Fig. 2C). Thus at different times after the surgery, the spatiotemporal patterns of $\mathrm{T} 1$ and $\mathrm{T} 4$ preganglionic projections from transplanted spinal cords were examined. $\mathrm{My}$ results indicate that preganglionic neurons from the donor spinal cord, even though they might be $1 \mathrm{~d}$ older than the host, still 
Figure 5. Sagittal sections showing development of $\mathrm{T} 1$ and T4 preganglionic projections. $A, \mathrm{~T} 1$ preganglionic axons begin to project into the sympathetic trunk at stage 27 (arrow). $B$, By stage $28, \mathrm{~T} 1$ axons have projected 3 segments rostrally, whereas $\mathrm{T} 4$ axons are just beginning to enter into the sympathetic trunk. Scale bar, $0.4 \mathrm{~mm}$.
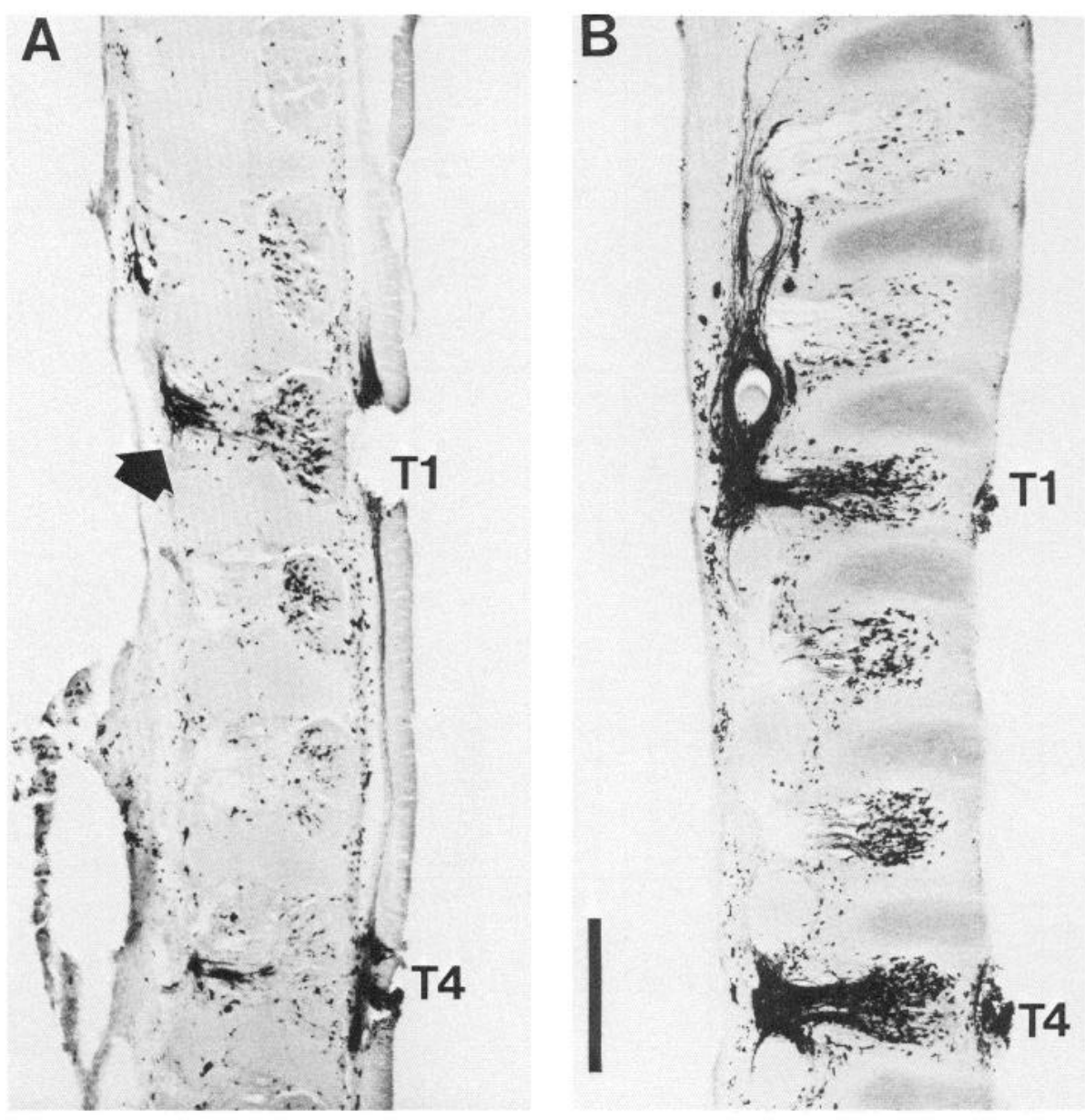

project according to the schedule of the host. Thus, when the T1-T4 spinal cord segment of a stage 18-19 donor was transplanted into a stage 13-14 host, donor preganglionic neurons at the $\mathrm{T} 1$ spinal level do not extend axons into the sympathetic trunk until the host develops to stage $27(n=15)$, the normal time at which $\mathrm{T} 1$ preganglionic axons project into the sympathetic trunk (see Fig. 5). By stage $28(n=11)$, donor T1 preganglionic neurons project 3 segments rostrally, whereas donor T4 preganglionics just begin to project into the sympathetic trunk (Fig. 9). These patterns of preganglionic projections are identical both to those of normal embryos and to embryos in which the T1-T4 spinal cord segment was rotated. Thus, the tissue environment not only influences the direction of preganglionic projections, but also appears to influence the timing of preganglionic outgrowth.

\section{Discussion}

A major finding of the present study is that the spatiotemporal patterns of sympathetic preganglionic projections are not determined intrinsically by the neurons' segmental origin in the spinal cord, but are influenced by the local environment along the path of their projections. This finding is consistent with results on the development of mammalian cortical projections from fetal cortical transplants. When cortical neurons are transplanted to different locations, they project in patterns that are appropriate to neurons of their new locations (Stanfield and O'Leary, 1985; O'Leary and Stanfield, 1989).

A question raised by the present finding is the nature of the local environmental cues. What kind of cells or cell products are there along the pathway of preganglionic axonal growth? The answers to these questions are not clear. However, in the innervation of skeletal muscles, there are some suggestions that Schwann cells along axonal pathways, themselves specified according to some positional values, may provide the directional cues for the outgrowth of motor axons. For example, there is some evidence that Schwann cells are located ahead of muscle nerves as they grow into the limb (Noakes and Bennett, 1987). In addition, depletion of Schwann cells through neural crest excision resulted in the inability of motor axons to enter into the limb (Carpenter and Hollyday, 1986; see, however, LanceJones and Yip, 1988). Whether Schwann cells are required for the guidance of sympathetic preganglionic projections is not certain. However, despite radical extirpation of the neural crest throughout cervical and thoracic levels, which should include Schwann cells as well, sympathetic preganglionic axons still maintain their normal trajectories and project to the regions where the ganglia would have been formed (Yip, 1987). It should be noted, however, that Schwann cells could still be present, due to their migration from segments adjacent to regions where the neural crest was removed. In addition, Schwann cells could also originate from the ventral neural tube (Weston, 1963; Loring et al., 1988).

An ultimate goal in the study of axonal guidance mechanisms is the identification of the molecular nature of the guidance cues. While there is no consensus on the exact identity of individual guidance molecules, several possible sources of molecular guid- 


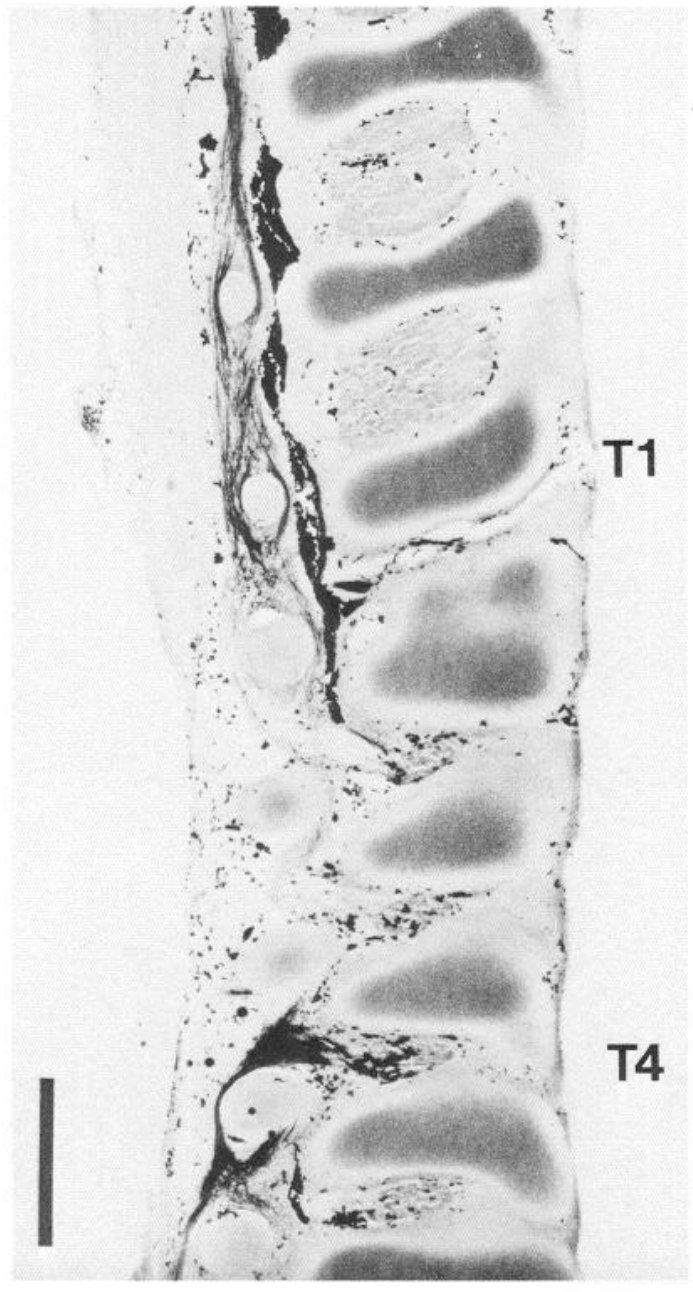

Figure 6. Sagittal section of a stage 30 embryo showing projections of $\mathrm{T} 1$ and T4 preganglionic neurons in spinal cord segment rotated $180^{\circ}$ around the anterior-posterior axis. T4 preganglionic neurons, translocated to the $\mathrm{T} 1 \mathrm{spinal}$ level project rostrally. $\mathrm{T} 1$ preganglionic neurons, translocated to the T4 spinal level project caudally. Scale bar, $0.4 \mathrm{~mm}$.

ance cues have been proposed. A possible source of guidance cues is a diffusible factor produced by neuronal targets or by tissues along axonal pathways that growing axons can detect and respond. A classic example of a diffusible factor showing such properties is the protein nerve growth factor (NGF). Under several experimental conditions, sympathetic and sensory neurons have been shown to respond to NGF gradients and extend axons toward the NGF source (Campenot, 1977; Menesini-Chen et al., 1978; Gunderson and Barret, 1980). More recently, it has been shown that the outgrowth of trigeminal axons toward their target is also chemotropic, even though the identity of the molecule is not known (Lumsden and Davis, 1983, 1986). In addition, in the development of the spinal cord, elongating commissural fibers appear to be guided by a diffusible factor produced by tissues in the floor plate region of the spinal cord (TessierLavigne et al., 1988). While the possibility that sympathetic preganglionic axons are guided by diffusible factors cannot be entirely eliminated, it is not likely, however, that they are guided by diffusible factors produced by their target tissues, since the absence of target tissues did not alter the projections of preganglionic axons (Yip, 1987). Another source of molecular cues that has been proposed are cell surface molecules. Several classes of cell surface molecules, including $\mathrm{N}$-CAM, $\mathrm{N}$-cadherin, $\mathrm{Ng}$ CAM/L1 have been shown to promote axon outgrowth (for reviews, see Lander, 1987; Dodd and Jessell, 1988; Jessell, 1988). The role of cell surface molecules on the outgrowth of sympathetic preganglionic axons is not known; work in progress, however, indicates that $\mathrm{N}-\mathrm{CAM}$ and $\mathrm{Ng}-\mathrm{CAM}$ are both present on preganglionic axons during outgrowth (unpublished observations).

A third source of guidance cues that could explain the present findings is the extracellular matrix (ECM) along the pathway of preganglionic axons. ECM molecules have been shown to promote neurite outgrowth in vitro, even though their role on neuronal outgrowth in vivo is unclear. Although the present study did not address whether ECM molecules are responsible for the guidance of sympathetic preganglionic axons, results from this study do suggest that there are factors in the pathway of preganglionic growth that can influence the outgrowth of preganglionic axons, a function that is consistent with ECM molecules (Lander, 1987; Sanes, 1989). Moreover, the rostral to caudal sequence in the normal development of preganglionic projections could reflect a spatiotemporal pattern in the expression of these molecules. Therefore, a working hypothesis for the specificity of preganglionic projections could be the sequential rostrocaudal expression of ECM or some axon outgrowth-promoting molecules along the pathway of preganglionic axonal growth. Because of the differential expression of these molecules in space and in time, neurons located at the T1 spinal level project rostrally in response to their presence along cervical levels. At a later time, these molecules are expressed more caudally, resulting in the caudal projection of neurons at the T4 spinal level.

This hypothesis, however, is built on the premise that translocated preganglionic neurons project in spatiotemporal patterns that are appropriate to neurons of their new segmental locations, and of course would be incorrect if translocated neurons are respecified. While respecification of translocated preganglionic neurons is a possibility, it does not appear likely here. In all my spinal cord rotation and transplantation experiments, including those from age-mismatched embryos, translocated preganglionic neurons at the $\mathrm{T} 1$ spinal level always project predominantly in the rostral direction and those at the T4 spinal level always project predominantly in the caudal direction, even though the operations were performed at widely different stages. More variations in this pattern would have been expected had respecification of preganglionic neurons occurred at some critical time. Additional results from this study, as well as from studies of other laboratories also indicate that the local environment has no effect on the identity of the translocated neurons when the transplantation was performed at stages of the present study. When segments of the thoracic spinal cord are transplanted to the cervical level, which is normally devoid of sympathetic preganglionic neurons, sympathetic preganglionic neurons retain their normal location around the central canal and project into the sympathetic chain. Similarly, motoneurons transplanted to different segmental locations maintain their original identity and connectivity (Narayanan and Hamburger, 1971; Lance-Jones and Landmesser, 1980).

My results on the response of sympathetic preganglionic projections to rotation of the spinal cord, however, appear to differ from those of somatic motor axons (Lance-Jones and Landmesser, 1980). Whereas displaced sympathetic preganglionic 

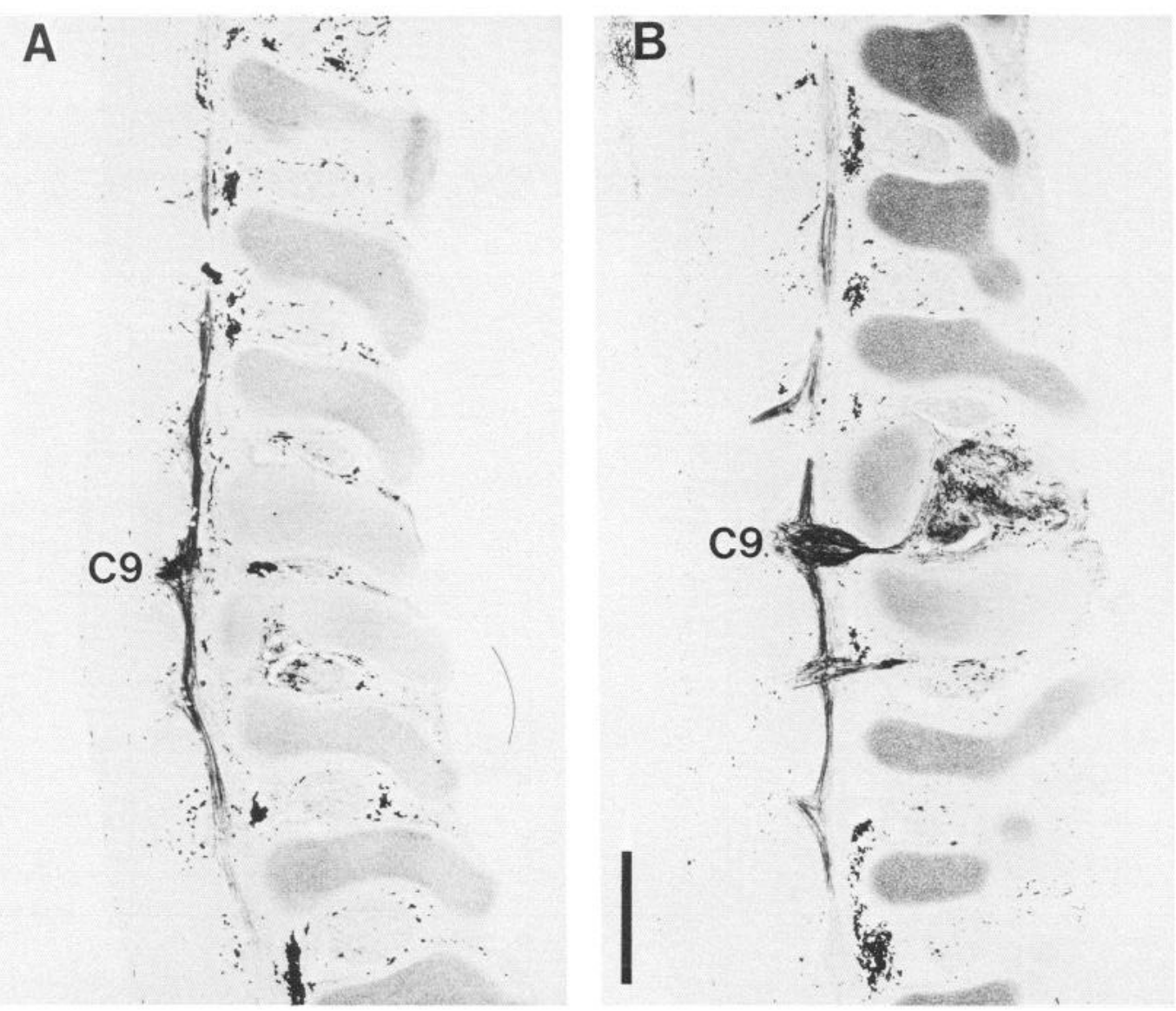

Figure 7. Sagittal sections from stage 30 embryos in which T1 $(A)$ and T4 $(B)$ preganglionic neurons have been translocated to the C9 spinal level. Instead of projecting respectively in their normal rostral or caudal direction, both $\mathrm{T} 1$ and $\mathrm{T} 4$ preganglionic neurons now project more or less equally in both directions. Scale bar, $0.4 \mathrm{~mm}$.

Figure 8. Sagittal sections showing early projections of T1 and T4 preganglionic neurons in spinal cord segments rotated $180^{\circ}$ around the anterior-posterior axis. $A$, At stage $27, \mathrm{~T} 4$ neurons translocated to the T1 spinal level project according to the schedule of their new location and are just starting to enter into the sympathetic trunk (arrow). $B$, At stage 28 , translocated T4 neurons project 3 segments rostrally while $\mathrm{T} 1$ neurons translocated to the T4 level are just beginning to enter the sympathetic trunk. Scale bar, $0.4 \mathrm{~mm}$.
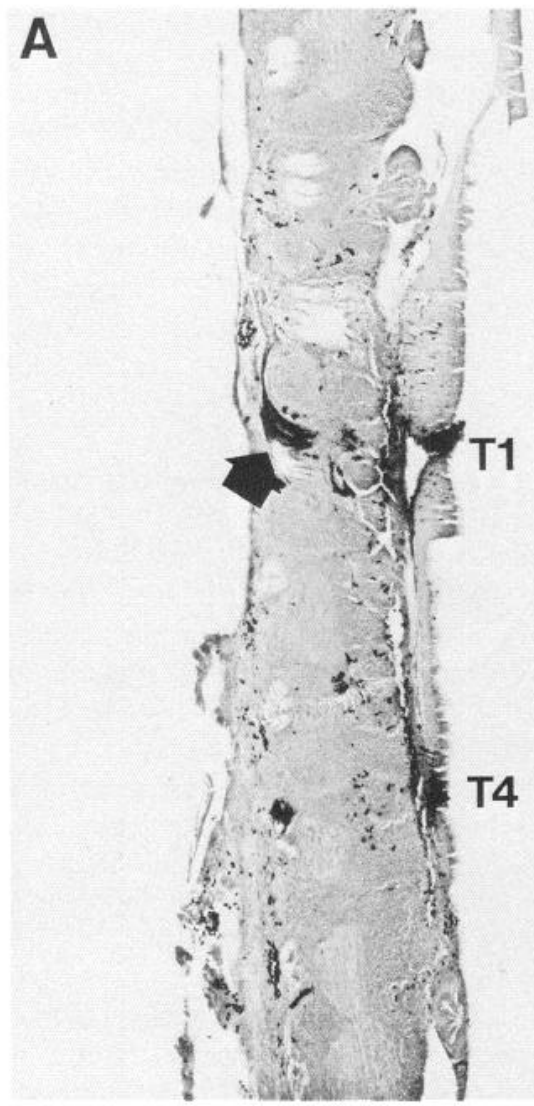

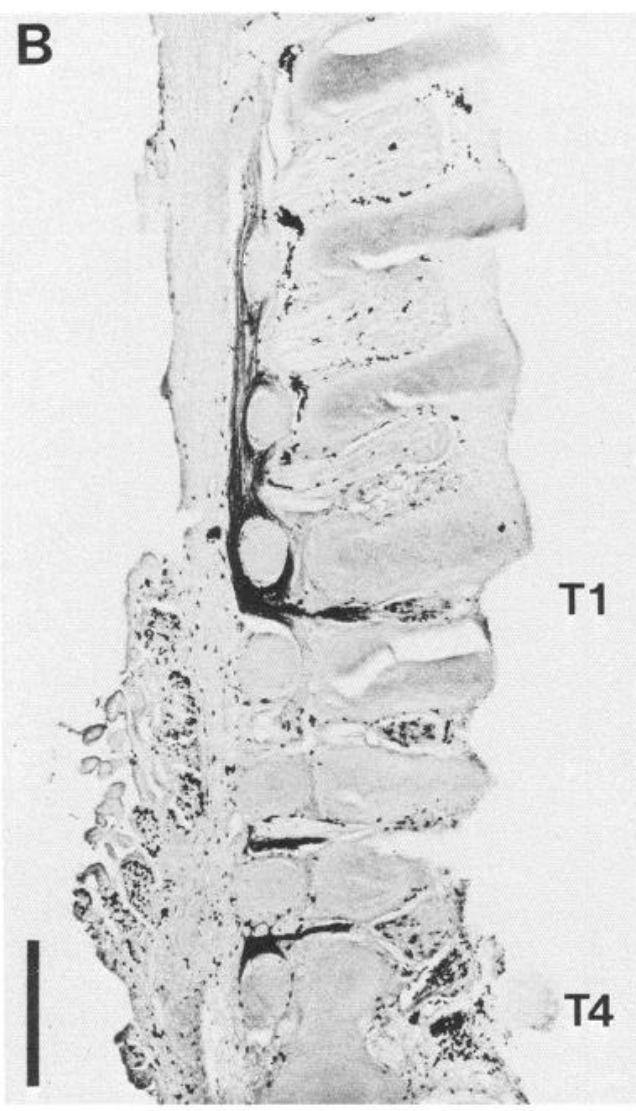



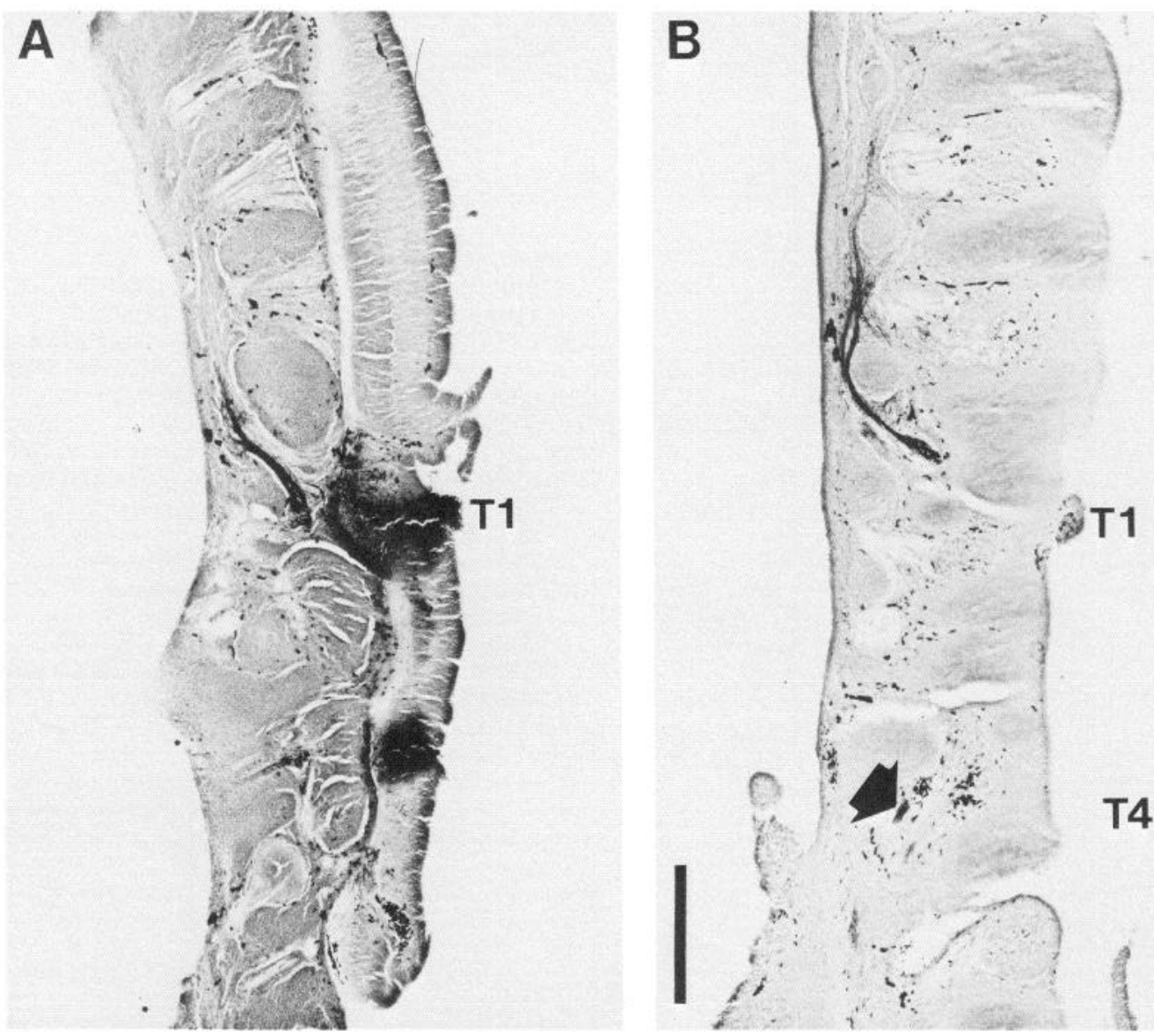

Figure 9. Sagittal sections showing projections of preganglionic neurons from transplants of older donor (stages 18-19) spinal cords into the sympathetic trunk of younger hosts (stages 13-14). At stage 27 of the host $(A)$, donor T1 preganglionics are just entering into the sympathetic trunk of the host, and by stage $28(B)$, they project 3 segments rostrally, whereas donor T4 preganglionics (arrow) are just beginning to enter into the sympathetic trunk. Scale bar, $0.4 \mathrm{~mm}$.

neurons project in directions that are appropriate to neurons of the new segmental locations, displaced motoneurons will return to their specified muscles. Without knowing the actual nature of axonal guidance cues in either system, it is not clear if a fundamental difference exists in axonal guidance mechanisms between these 2 systems. What is clear is that in both systems, axonal guidance appears to be independent of target cues but appears to be dependent on local environmental cues along the pathways of their axonal growth (Tosney and Landmesser, 1984).

While spinal cord rotation studies suggest that local environmental cues along the pathway of preganglionic growth are crucial in the guidance of preganglionic axons, a complication in the T1-T4 spinal cord rotation experiment is that not only are the anterior-posterior positions of T1 and T4 neurons reversed, but the laterality of the neurons in the spinal cord is also changed. Thus, for example, if T4 preganglionic neurons located on the left side of the spinal cord are specified to turn left upon entry of the sympathetic chain, when translocated to the T1 spinal level of the right side they would project rostrally. Neuronal specification, however, does not seem to be the case here. When single segments of the $\mathrm{T} 1$ or $\mathrm{T} 4$ spinal cord segment were transplanted to a cervical level, there is no distinction in the projection pattern between $\mathrm{T} 1$ and $\mathrm{T} 4$ preganglionic neurons, indicating that specific patterns of preganglionic outgrowth are determined by the local environment, and not by the segmental identity of preganglionic populations.
In summary, this study indicates that preganglionic projections are directed by local cues. This is similar to findings in the development of cortical projections in the mammal (Stanfield and O'Leary, 1985; O'Leary and Stanfield, 1989), motoneuron outgrowth in the chick (Lance-Jones and Landmesser, 1980; Hollyday, 1981), and sensory projections in insects (Blair and Palka, 1985; Blair et al., 1985; Lefcort and Bentley, 1987). In addition, this study also shows that the timing of preganglionic axonal outgrowth is influenced by local cues. Taken together, one might conclude that preganglionic axonal outgrowth is guided by local cues which may appear in a controlled spatiotemporal manner. Knowing the location and timing of the expression of guidance cues is crucial to their ultimate identification.

\section{References}

Adam SC (1977) Technical considerations on the use of HRP as a neuronal marker. Neuroscience 2:141-146.

Blair SS, Palka J (1985) Axon guidance in cultured wing discs and disc fragments of Drosophila. Dev Biol 108:411-419.

Blair SS, Murray MA, Palka J (1985) Axon guidance in cultured epithelial fragments at Drosophila wing. Nature 315:406-409.

Campenot RB (1977) Local control of neurite development by nerve growth factor. Proc Natl Acad Sci USA 74:4516-4519.

Carpenter EM, Hollyday M (1986) Defective innervation of chick limbs in the absence of presumptive Schwann cells. Soc Neurosci Abstr 12:1210. 
Dodd J, Jessell TM (1988) Axon guidance and the patterning of neuronal connections in vertebrates. Science 242:692-699.

Eisen JS, Myers PZ, Westerfield M (1986) Pathway selection by growth cones of identified motoneurones in live zebra fish embryos. Nature 230:269-271.

Gundersen RW, Barrett JN (1979) Neuronal chemotaxis: chick dorsalroot axons turn toward high concentration of nerve growth factor. Science 206:1079-1080.

Hamburger V, Hamilton HL (1951) A series of normal stages in the development of the chick embryo. J Morphol 88:49-92.

Hanker JS, Yates PE, Metz CB, Rustioni A (1977) A new, specific, sensitive and non-carcinogenic reagent for the demonstration of horseradish peroxidase. J Histochem 9:789-792.

Hollyday M (1981) Rules of motor innervation in chick embryos with supernumerary limbs. J Comp Neurol 202:439-465.

Jessel TM (1988) Adhesion molecules and the hierarchy of neural development. Neuron 1:3-13.

Lance-Jones C, Landmesser L (1980) Motoneurone projection patterns in chick hind limb following partial reversals of the spinal cord. J Physiol (Lond) 302:581-602.

Lance-Jones C, Yip JW (1988) The effect of neural crest deletions on the development of specific motoneuron projection in the embryonic chick hindlimb. Soc Neurosci Abstr 14:451.

Lander A (1987) Molecules that make axons grow. Mol Neurobiol 1: 213-245.

Landmesser L (1978) The distribution of motoneurones supplying chick hind limb muscles. J Physiol (Lond) 284:371-389.

Langley JN (1904) On the sympathetic system of birds and on the muscles which move the feathers. J Physiol (Lond) 30:221-252.

LeDouarin N (1982) The neural crest. Cambridge, UK: Cambridge University Press.

Lefcort F, Bentley D (1987) Pathfinding by pioneer neurons in isolated, opened and mesoderm-free limb buds of embryonic grasshoppers. Dev Biol 1 19:466-480.

Levi-Montalcini $R$ (1950) The origin and development of the visceral system in the spinal cord of the chick embryo. J Morphol 81:253282.

Loring JF, Barker DL, Erickson CA (1988) Migration and differentiation of neural crest and ventral neural tube cells in vitro: implications for in vitro and in vivo studies of the neural crest. J Neurosci 8:1001-1015.
Lumsden AGS, Davies AM (1983) Earliest sensory nerve fibres are guided to peripheral targets by attractants other than nerve growth factor. Nature 306:786-788.

Lumsden AGS, Davies AM (1986) Chemotrophic effect at specific target epithelium in the developing mammalian nervous system. $\mathrm{Na}$ ture 323:538-539.

Menesini-Chen MG, Chen JS, Levi-Montalcini R (1978) Sympathetic nerve fibers ingrowth in the central nervous system of neonatal rodent upon intracerebral NGF injection. Arch Ital Biol 116:53-84.

Narayanan $\mathrm{CH}$, Hamburger V (1971) Motility in chick embryos with substitution of lumbosacral by brachial and brachial by lumbosacral spinal cord segments. J Exp Zool 178:415-432.

Noakes PG, Bennett MR (1987) Growth of axons into developing muscles of the chick forelimb is preceded by cells that stain with Schwann cell antibodies. J Comp Neurol 259:330-347.

O'Leary DDM, Stanfield BB (1989) Selective elimination of axons extended by developing cortical neurons is dependent on regional locale: experiments utilizing fetal cortical transplants. J Neurosci 9 : $2230-2246$.

Sanes JR (1989) Extracellular matrix molecules that influence neural development. Annu Rev Neurosci 12:491-516.

Stanfield BB, O'Leary DDM (1985) Fetal occipital cortical neurons transplanted to the rostral cortex can extend and maintain a pyramidal tract axon. Nature 313:135-137.

Terni T (1924) Ricerche anatomiche sul sistema nervoso autonomo degli Uccelli. Arch Ital Anat Embriol 20:433-510.

Tessier-Lavigne M, Placzek M, Lumsden AGS, Dodd J, Jessell TM (1988) Chemotrophic guidance of developing axons in the mammalian central nervous system. Nature 336:775-778.

Tosney KW, Landmesser L (1984) Pattern and specificity of axonal outgrowth following varying degrees of chick limb bud ablation. $\mathrm{J}$ Neurosci 4:2518-2527.

Weston JA (1963) A radioautographic analysis of the migration and localization of trunk neural crest cells in the chick. Dev Biol 6:279310.

Yip JW (1986) Specific innervation of neurons in the paravertebral sympathetic ganglia of the chick. J Neurosci 6:3459-3464.

Yip JW (1987) Target cues are not required for the guidance of sympathetic preganglionic axons. Dev Brain Res 32:155-159. 